Revue pluridisciplinaire d'études médiévales

\title{
Rôles et fonctions des archaïsmes dans les représentations vestimentaires au Moyen Âge
}

Tina Anderlini

\section{OpenEdition}

\section{Journals}

Édition électronique

URL : http://journals.openedition.org/questes/5151

DOI : 10.4000/questes.5151

ISSN : 2109-9472

Éditeur

Les Amis de Questes

Édition imprimée

Date de publication : 28 février 2019

Pagination : 54-79

ISSN : 2102-7188

\section{Référence électronique}

Tina Anderlini, «Rôles et fonctions des archaïsmes dans les représentations vestimentaires au

Moyen Âge », Questes [En ligne], 40 | 2019, mis en ligne le 19 avril 2019, consulté le 22 avril 2019. URL http://journals.openedition.org/questes/5151 ; DOI : 10.4000/questes.5151 


\title{
Rôles et fonctions des archaïsmes dans les représentations vestimentaires au Moyen Âge
}

\author{
Tina ANDERLINI \\ Chercheuse associée au CESCM, Université de Poitiers
}

Avec le costume préhistorique, le costume médiéval est l'un des moins étudiés. En dehors des vêtements conservés comme reliques, l'archéologie a fourni peu de tissus médiévaux, et dans tous les cas, ce mobilier est souvent très altéré ou fragmentaire. De plus, la perception que nous en avons est généralement biaisée par les reconstitutions fantaisistes qui se sont multipliées depuis le XIX ${ }^{\mathrm{e}}$ siècle. Ainsi, avant d'essayer de comprendre le costume médiéval, le chercheur peut en avoir certaines idées qui se révèlent totalement erronées.

Cette rareté des témoignages matériels oblige à se tourner vers les images pour reconstituer les habitudes vestimentaires médiévales. Ces images sont nombreuses, souvent passionnantes à analyser, mais leur étude impose de nombreuses précautions : l'image médiévale est soumise à certaines conventions qui, bien souvent, remontent à l'Antiquité, à la période paléochrétienne ou à l'art byzantin. Après avoir écarté les reconstitutions fautives ou plus ou moins fantasmées au profit des images contemporaines, le chercheur se trouve dans l'obligation de devoir trier les représentations médiévales elles-mêmes. La lecture de ces dernières nécessite une méthodologie rigoureuse. En effet, une même image médiévale peut tout à la fois représenter des éléments de costumes portés à l'époque de production de l'œuvre, des vêtements appartenant à un passé réel mais qui ne sont alors plus portés, des tentatives de reconstituer des costumes relevant d'un passé mal compris et des accoutrements plus ou moins fantaisistes. 
On peut néanmoins essayer de restituer les différents costumes en usage au cours du millénaire médiéval en croisant les sources. Aux images trop souvent trompeuses, il faut confronter ce que nous apprennent la littérature, les textes législatifs, les inventaires, les testaments et les quelques pièces de mobilier archéologique. L'ensemble doit être analysé avec soin pour démêler le vrai, le vraisemblable et le «faux», qui peut prendre plusieurs formes. Par « faux », nous entendons ici un costume obsolète, désuet ou anachronique par rapport à l'époque de production ou au contexte historique représenté. Souvent, c'est le passage du temps ou un décalage temporel qui explique les différences entre le costume représenté et celui porté au moment où l'œuvre est produite. L'omniprésence de costumes désuets, obsolètes ou anachroniques dans les représentations médiévales rend nécessaires une bonne connaissance des évolutions de la mode médiévale, ainsi qu'un effort de confrontation constant avec les autres sources.

Au Moyen Âge, le costume est loin d'être anodin. Il est un indicateur de rang social, de bon goût, d'éducation, ou bien de vulgarité, de pingrerie ${ }^{1}$. Il est utilisé pour distinguer le bon chrétien des exclus, comme les Juifs ou les prostituées $^{2}$. L'habit fait le moine, quoi qu'en dise le proverbe ${ }^{3}$. Et il est important pour la société médiévale qu'il en soit ainsi. Le vêtement est alors la principale dépense somptuaire visible, les autres dépenses comme les tournois et les banquets étant limitées dans l'espace et le temps. En revanche, le costume participe puissamment de l'image que l'on veut donner de soi aux autres, à l'extérieur et dans son intérieur. La littérature laisse apparaître une hiérarchie des

\footnotetext{
${ }^{1}$ La littérature concernant cet aspect est abondante. Nous nous contenterons de renvoyer à cet ouvrage récent : Sarah-Grace Heller, Fashion in Medieval France, Cambridge, D.S. Brewer, 2007.

${ }^{2}$ Voir à ce sujet, entre autres, Michel Pastoureau, L'Étoffe du Diable, Paris, Éditions du Seuil, 1991, et Bernhard Blumenkranz, Le Juif médiéval au miroir de l'art chrétien, Paris, Études augustiniennes, 1966.

${ }^{3}$ Voir Fanny Oudin, «L'habit fait-il le moine? Quelques réflexions autour des proverbes

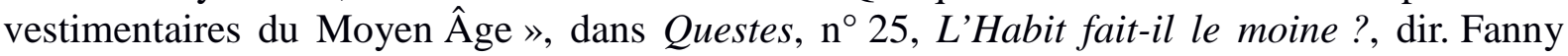
Oudin, 2013.
} 
vêtements, que l'on met ou ôte selon les circonstances et selon le rang des personnages qui nous entourent ${ }^{4}$. Ainsi, le vêtement est resté jusqu'à la fin du $\mathrm{XX}^{\mathrm{e}}$ siècle l'un des principaux symboles du statut social.

Ces considérations nous paraissent nécessaires pour mettre en avant les difficultés méthodologiques et les enjeux des études sur le costume. Ces difficultés et enjeux étant posés, nous allons maintenant considérer quels vêtements peuvent être qualifiés d'obsolètes, puis nous tenterons de comprendre pourquoi les médiévaux et les artistes du Moyen Âge ont choisi de porter des habits désuets ou de figurer, par anachronisme, des vêtements d'un autre temps.

Plusieurs tendances se dessinent. L'obsolète nous paraît se manifester uniquement dans la représentation de la réalité et y revêtir un rôle précis. De leur côté, le désuet et l'anachronique correspondraient à la fois à une réalité historique et à sa représentation. Dans tous les cas, l'usage de costumes anciens répond à des besoins spécifiques, à des circonstances précises. Car plus que des règles générales, ce sont des contextes particuliers qui importent. Et ceci est autant valable pour les œuvres d'art sur tous supports que pour les vêtements qui furent historiquement portés.

\section{Accessoires et vêtements obsolètes}

Nous définirons ici comme obsolète un vêtement ou une habitude vestimentaire qui, anciennement à la mode, n'est plus en usage mais continue à figurer dans des contextes précis sur certaines illustrations. Comme le montrent les exemples du touret et du vêtement long, le vêtement obsolète semble utilisé comme une convention picturale figurant l'altérité.

\footnotetext{
${ }^{4}$ Ceci est particulièrement visible dans les romans de Jean Renart (ou Renaut), écrits autour de 1200, Galeran de Bretagne et L'Escoufle (Jean Renart, L'Escoufle, roman d'aventure. Nouvelle édition d'après le manuscrit 6565 de la Bibliothèque de l'Arsenal, éd. Franklin Sweetser, Genève, Droz, 1974. L'Escoufle, roman d'aventures, trad. Alexandre Micha, Paris, Champion, 1992. Galeran de Bretagne, éd. bilingue, trad. et notes par Jean Dufournet, Paris, Champion, 2009).
} 


\section{Représenter le touret, un accessoire de mode devenu obsolète}

Le touret est le sommet (au propre comme au figuré) de l'élégance féminine du XIII siècle. La coiffe disparaît durant le premier quart du $\mathrm{XIV}^{\mathrm{e}}$ siècle, mais elle est toutefois représentée dans certaines images du Heilsspiegel ${ }^{5}$, datant de la seconde moitié du $\mathrm{XIV}^{\mathrm{e}}$ siècle. Ce manuscrit utilise l'un des principes fondamentaux de l'image médiévale: la typologie. Pour simplifier schématiquement, ce procédé consiste à mettre en rapport des scènes de l'Ancien et du Nouveau Testament, les premières préfigurant les secondes. Mises en miroir, scènes vétérotestamentaires et néotestamentaires constituent un couple type-antitype. Or, dans le Heilsspiegel, les tourets ne sont portés que par des personnages féminins de l'Ancien Testament (image 1). Le touret, coiffe totalement démodée, est devenu le marqueur d'une époque révolue, mais aussi d'un ailleurs, l'un et l'autre semblant se confondre. Il cohabite avec des vêtements contemporains du contexte de création de l'œuvre, et des marqueurs vestimentaires relevant de traditions iconographiques. Phénomène de mode alors obsolète, il sert à marquer l'éloignement spatial et temporel des personnages figurés. En outre, il est représenté d'une manière jamais observée auparavant. La coiffe, moins d'un siècle après sa disparition, est mal comprise. Cette incompréhension se retrouve sur un gisant néerlandais, restauré au $\mathrm{XVI}^{\mathrm{e}}$ siècle. Ce gisant se trouve dans l'église abbatiale de Roermond ; c'est celui du duc Gérard III de Gueldre et de Marguerite de Brabant, morts en 1229 et 1231. Les costumes du XIII ${ }^{\mathrm{e}}$ siècle sont totalement incompris et le touret a une structure unique en son genre : trop de plis à la barbette et une décoration dorée ajoutée à un accessoire traditionnellement totalement blanc en Europe du Nord. Le

\footnotetext{
${ }^{5}$ Landesbibliothek Darmstadt, Ms 2505, vers 1360. L'intégralité du manuscrit est publiée dans Heilsspiegel. Die Bilder des mittelalterliche Erbauungsbuches Speculum humanae salvationis, avec des commentaires de Horst Appuhn, Die bibliophilen Taschenbücher, $\mathrm{n}^{\circ}$ 267, Dortmund, Harrenberg, 1981.
} 
restaurateur a complètement réinventé la mode du passé. La mauvaise restitution devient ainsi l'indice d'une intervention ultérieure ${ }^{6}$.

\section{Le vêtement long et les manches au $\mathrm{XV}^{\mathrm{e}}$ siècle}

Si les coiffes peuvent servir à figurer le temps qui s'est écoulé, il en est de même pour les vêtements proprement dits et, à ce titre, l'évolution des longueurs peut aussi être un marqueur. Il est très difficile d'aborder cet aspect car vêtements longs et courts cohabitent à partir du $\mathrm{XIV}^{\mathrm{e}}$ siècle. Le vêtement long, prépondérant depuis le XII siècle, laisse progressivement place au vêtement court, devenu omniprésent au $\mathrm{XV}^{\mathrm{e}}$ siècle. À cette période, il apparaît comme un marqueur de modernité, le vêtement long étant alors réservé à des usages bien particuliers.

Sur le modèle des œuvres usant de la typologie, celles souhaitant opposer des catégories différentes de personnages affublent ces derniers de vêtements courts et longs. C'est notamment le cas pour distinguer Chrétiens et Juifs, nouveauté et ancienneté vestimentaires reflétant celles des religions concernées ${ }^{7}$. Les païens ont pour leur part droit à une tenue qui relève davantage d'une fantaisie exotique, reflétant un monde hors du Livre. Chaque forme de vêtement est ainsi associée à une catégorie de personnages plus ou moins éloignés de la réalité de l'Occident du milieu du XV siècle (image 2). En outre, ces robes longues sont dotées de manches amples, rappelant les houppelandes à la mode vers 1400 mais ensuite passées de mode chez les hommes. Le vêtement obsolète et, en particulier, un de ses détails deviennent pour les auteurs chrétiens la

\footnotetext{
${ }^{6}$ Il est possible que le tombeau ne soit pas le fruit de la restauration d'une œuvre endommagée, mais une recréation complète en ce qui concerne les personnages. Plusieurs théories se sont succédé : on pourra lire à ce sujet Jitske Jasperse, « Duke Charles of Guelders and the "Restoration" of the Tomb Monument of Gerard IV and Margaret in the Roermond Minster », dans Medieval art in the Northern Netherlands, dir. Claudine Chavannes-Mazel et Anne-Maria van Egmond, Utrecht, New facts and features, 2014, p. 174-187 (un grand merci à Magali de la Reina pour m'avoir procuré cet article).

${ }^{7}$ Rational des divins offices, Bibliothèque municipale, Beaune, ms 21, fol. $149, \mathrm{XV}^{\mathrm{e}}$ siècle. Merci à Pamela Nourrigeon de m'avoir fait découvrir cette illustration.
} 
marque d'une religion qu'ils considèrent comme obsolète depuis la venue du Messie.

\section{Le costume désuet : des connotations antithétiques}

Le cas des vêtements courts et longs nous conduit à aborder la question des costumes désuets. En effet, si le vêtement long devient obsolète et disparaît de la vie quotidienne au $\mathrm{XV}^{\mathrm{e}}$ siècle, son usage se perpétue dans certaines situations précises. De manière paradoxale, le caractère désuet de ce type de vêtement n'est pas systématiquement dépréciatif. Comme nous allons le voir, il peut être connoté tout aussi bien positivement que négativement.

\section{Un aspect suranné vite tourné en dérision}

Si le vêtement obsolète ne se porte plus, le désuet, lui, persiste dans l'usage mais se teinte d'une coloration surannée. Dans les représentations littéraires ou visuelles, un costume désuet peut caractériser un personnage qui ne prend pas soin de lui ou dont le caractère est maussade. Un bon exemple en est le triste et jaloux Archambault du roman de Flamenca ${ }^{8}$. Personnage par ailleurs trompé, puisque son épouse Flamenca lui préfère le jeune et beau Guillaume, vêtu à la dernière mode, et dont le costume comme le comportement répondent aux canons de la culture courtoise ${ }^{9}$. Dans le roman courtois, le vêtement désuet est également la marque d'une certaine avarice. Pourtant, certaines sources écrites, comme la biographie de saint Louis par Joinville, nous montrent que les vêtements pouvaient se transmettre de génération en génération ${ }^{10}$.

\footnotetext{
${ }^{8}$ Flamenca. Texte édité d'après le manuscrit unique de Carcassonne, éd. François Zufferey, trad. Valérie Fasseur, Paris, Le Livre de Poche, coll. «Lettres gothiques », 2014.

${ }^{9}$ Sarah-Grace Heller, Fashion in Medieval France, op. cit., p. 68-69.

${ }^{10}$ Jean de Joinville, Vie de Saint Louis, éd. et trad. Jacques Monfrin, Paris, Garnier, 1995, p. 164-165.
} 
Toutefois, si le vêtement désuet est parfois présenté négativement comme l'inverse de la galanterie et de la coquetterie, il est considéré par d'autres acteurs et dans d'autres contextes comme une marque de respectabilité.

\section{Un symbole de respectabilité}

Ces utilisations antithétiques du vêtement ne doivent pas nous surprendre et correspondent parfaitement aux principes de l'image médiévale ${ }^{11}$. Selon les contextes, tout élément d'une image peut être soit positif, soit négatif, soit neutre. Il appartient à l'historien de l'art de tenter de démêler les fils de la toile pour retrouver l'information donnée par l'auteur, sans oublier les dangers d'une telle exégèse. La connotation positive de la désuétude d'un costume nous semble particulièrement intéressante. En effet, si le costume désuet peut être tourné en dérision, les médiévaux tendent également à considérer le costume venant du passé comme un signe de respectabilité ${ }^{12}$. Quand il est honorable, il peut symboliser la respectabilité de la famille à laquelle on appartient ou justifier le rang de celui qui le porte. Dans ce cas, il est le témoin d'une histoire familiale dont on est fier. Le vêtement appartenant à une époque révolue devient aussi un symbole de pouvoir. Le costume royal lui-même répond à des conventions pour les images et pour la représentation. Ainsi le roi s'habille de long, même lorsque la mode est au court. Jeune, Charles V suivait la mode, mais il adopta dès son couronnement une garde-robe plus traditionnelle ${ }^{13}$. Charles VI fut quant à lui le premier roi «ouvertement» à la mode au cours de son règne ${ }^{14}$. Mais modes

\footnotetext{
${ }^{11}$ Même l'utilisation thèse-antithèse n'est pas théorisée, comme le souligne Jean Wirth dans L'Image médiévale. Naissance et développement $\left(V I^{e}-X V^{e}\right.$ siècle), Paris, Méridiens Klincksieck, 1989, p. 232-236.

${ }^{12} \mathrm{Qu}$ 'on ne soit pas surpris par l'aspect à la fois positif et négatif d'un même objet. Toute la symbolique médiévale fonctionne ainsi. On en trouvera de multiples exemples dans les études de Michel Pastoureau ou de Jean Wirth, pour ne citer qu'eux.

13 «Le nouveau roi délaisse l'habit court des chevaliers pour prendre la robe longue des clercs et des officiers en droit » (Claude Gauvard, La France au Moyen Âge du $V^{e}$ au XV siècle, Paris, PUF, 1996, p. 403).

${ }^{14}$ Françoise Piponnier, «Une révolution dans le costume masculin au XIV siècle », dans $L e$ Vêtement. Histoire, archéologie et symbolique vestimentaires au Moyen Âge, dir. Michel
} 
longue et courte cohabitent. La première, remontant au XIII ${ }^{\mathrm{e}}$ siècle, est une marque de respectabilité. La seconde est l'apanage de la jeunesse dorée de l'époque. Même si un roi se permet quelques fantaisies vestimentaires, les représentations publiques d'un événement tentent de le dissimuler. Lorsque Charles V le Sage reçoit en janvier 1378 son oncle, l'empereur Charles IV, il porte un chapeau à bec très à la mode. Pourtant, la représentation figurée de cet événement dans les Grandes Chroniques de France n'en tient pas compte ${ }^{15}$ : le roi y est montré paré de sa couronne, un antique symbole de pouvoir qui permet de le reconnaître ${ }^{16}$. C'est que, dans les mentalités médiévales, l'autorité est associée à la tradition, y compris vestimentaire.

En effet, le costume cérémoniel se réfère au passé. Les dames de la noblesse, jusqu'à une date très avancée (le début du XVI $\mathrm{e}^{\mathrm{e}}$ siècle) portent le surcot lors des grandes occasions. Ce vêtement ample est apparu à la fin du XII siècle, en réaction à la mode fortement érotisée qui se développe à l'extrême fin du $\mathrm{XI}^{\mathrm{e}}$ siècle ${ }^{17}$. Il cache les formes masculines comme féminines. Pour les femmes, il va évoluer au cours du XIV ${ }^{\mathrm{e}}$ siècle. La mode est alors aux tenues serrées sur le torse, mettant la taille et les hanches en valeur. Le surcot s'ouvre et prend le surnom de «surcot à porte (ou fenêtre) d'enfer ». Cette tenue est revêtue par les reines comme Jeanne de Bourbon (image 3) et par d'autres nobles dames, telle Jeanne de Hangest (image 4), morte en 1514, dans les grandes occasions ou pour

Pastoureau, Paris, Éditions du Léopard d'Or, coll. «Cahiers du Léopard d'Or », n 1, 1989, p. 225-253, voir p. 233.

${ }^{15}$ Grandes Chroniques de France, Paris, Bibliothèque Nationale, fr. 2813, fol. 470v, Paris, vers $1375-1379$.

${ }^{16}$ Odile Blanc, Parades et Parures. L'invention du corps de mode à la fin du Moyen Âge, Paris, Gallimard, 1997, p. 13. Voir l'image 1, p. 74.

${ }^{17}$ Voir par exemple Frédérique Lachaud, «La critique du vêtement et du soin des apparences », dans Le Corps et sa parure, dir. Jean Wirth, Micrologus XV, Firenze, SismelEdizioni del Galuzzo, 2007 ; Sarah-Grace Heller, Fashion in Medieval France, op. cit.; Étienne de Bourbon (119.?-1261 ?), Anecdotes historiques, légendes et apologues tirés du Recueil inédit d'Étienne de Bourbon, dominicain du XIII siècle, éd. A. Lecoy de La Marche, Paris, Renouard, 1877 ; Henri Platelle, «Le Problème du scandale : les nouvelles modes masculines aux XI $\mathrm{XI}^{\mathrm{e}}$ et XII ${ }^{\mathrm{e}}$ siècles », Revue belge de philologie et d'histoire, t. 53, fasc. 4, 1975, p. 1071-1096. 
l'éternité sur les gisants. Cottehardies, houppelandes, robes à tassel se succèdent dans la garde-robe quotidienne de la noblesse. Pourtant, l'antique surcot persiste, s'adapte, en dévoilant les hanches mais aussi en s'agrémentant de boutons inutiles, en mettant en évidence les fourrures. Le surcot, vêtement mixte et pudique au XIII ${ }^{\mathrm{e}}$ siècle, est désormais un incontournable de la tenue cérémonielle féminine, glorifiant le corps. Le costume masculin subit également l'impact de ces évolutions. Né au XIII siècle, ce phénomène de mode conduit aux transformations de certains costumes, comme ceux des docteurs et des membres du parlement. Un détail, les petits plis qu'on peut voir sur la plaque funéraire de l'architecte rémois Libergier (image 5), va survivre bien plus longtemps que le surcot à porte d'enfer, et se voit toujours sur les manches des juges, avocats et autres érudits, dans certaines circonstances ${ }^{18}$.

Les éléments désuets du costume apparaissent bien, dans certains cas, comme des marques d'autorité, des signes sociaux très forts. Cette utilisation de tenues désuètes dans quelques circonstances bien précises et cérémonielles ne s'arrête pas avec le Moyen Âge. Un vêtement désuet en remplace un autre. On peut s'interroger sur la survie et les raisons de l'abandon final de ces vêtements désuets dans l'image ${ }^{19}$. Il apparaît clairement que le costume ancien, marque d'une tradition, est perçu comme rassurant, comme le tenant d'une continuité. À quel moment un vêtement désuet, mais qui est porteur de sens ou de respectabilité, devient-il suranné ou obsolète? Cette évolution advient-elle lorsqu'un pouvoir qui en a fait son attribut apparaît comme affaibli ou en déclin? Dans des circonstances particulières, la modernisation d'un costume

\footnotetext{
${ }^{18}$ Ces plis figurent au départ sur les manches d'un vêtement d'extérieur, la chape, porté par les nobles et les bourgeois, et aussi tenue de voyage. La chape deviendra la robe des juges et la toge des docteurs.

${ }^{19}$ On peut penser à un exemple contemporain : le costume porté par les présidents de la République sur leur photographie officielle. Jusqu'à Georges Pompidou (mort en 1974), les présidents portaient le frac, une tenue venue du passé. Son successeur, Valéry Giscard d'Estaing, l'abandonna pour le costume de ville. Ceci fut interprété comme une marque de modernité et une volonté de réforme. La photographie officielle de Giscard était, dans toute sa composition, en rupture totale avec celle de ses prédécesseurs.
} 
solennel indique la volonté de son porteur d'apparaître plus en adéquation avec son temps. En cela, il est souvent révélateur d'une volonté de réforme.

Les vêtements présentant des caractères désuets et connotant une certaine respectabilité permettent de légitimer celui qui en use, en le liant à l'histoire d'une institution vénérable par son ancienneté. Ainsi, les plis des manches des magistrats remontent au XIII ${ }^{\mathrm{e}}$ siècle. Même s'ils ont évolué avec le temps, la persistance de ce caractère vestimentaire contribue à conférer à la Justice un caractère respectable, intemporel, éternel.

Tout comme le fait d'acheter un château ou un titre de noblesse, se parer d'un tel costume aux caractères désuets peut être un moyen de s'offrir une légitimité.

\section{Les anachronismes dans les représentations de costumes}

Les costumes anachroniques par rapport au contexte de leurs porteurs sont nombreux dans les représentations picturales médiévales. Les textes copiés durant plusieurs siècles sont tout particulièrement concernés. En effet, lorsque les documents sont copiés au cours du temps, les enlumineurs continuent à peindre les costumes attribués aux personnages au moment de la réalisation des premiers manuscrits. Les copies successives continuent à reproduire les costumes d'origine, souvent antiques ou carolingiens, en les dénaturant, en les ornant à outrance (une convention graphique liant ce qui est ancien et respectable à ce qui est ouvragé et de valeur) ou en y ajoutant des éléments nouveaux. Les contemporains de ces images plus récentes comprenaient-ils ces détails vestimentaires venus du passé ? Des pièces de Térence sont reproduites par trois fois, d'après un manuscrit original antique disparu ${ }^{20}$. Les costumes $\mathrm{y}$ sont quasi identiques, mais la copie la plus récente se distingue par la richesse

\footnotetext{
${ }^{20}$ Rome, Bibliothèque Vaticane, Vat. lat. 3868, IX ${ }^{\mathrm{e}}$ siècle. Paris, Bibliothèque nationale de France, Latin 7899, IX ${ }^{\mathrm{e}}$ siècle. Oxford, Bodleian Library, Ms. Auct. F.2.13, milieu du XII ${ }^{\mathrm{e}}$ siècle.
} 
des décorations. Le Psautier d'Utrecht (image 6), chef-d'œuvre du IX siècle issu de l'École de Reims, a connu, lors de son passage en Angleterre, au moins trois copies. Chaque époque a ajouté des éléments nouveaux, tout en conservant des détails originaux ${ }^{21}$.

Toutefois, les costumes des personnages les plus sacrés sont ceux qui ont le moins changé. Dans les représentations de scènes bibliques, le costume à l'antique identifie immédiatement les individus les plus respectables et nobles ${ }^{22}$. On reconnaît ainsi Moïse grâce à sa toge dans les fresques de Botticelli sur les murs de la chapelle Sixtine, ou les apôtres, vêtus de la même manière, dans les peintures de Giotto. Encore une fois, le vêtement à l'ancienne indique un statut supérieur.

\section{Une sémiologie graphique partagée par tout l'Occident médiéval}

On constate également que certains anachronismes dans les costumes représentés constituent de véritables conventions visuelles permettant d'identifier des personnages précis. Le plus souvent, ces conventions sont communes à tout l'Occident chrétien. Sur toutes les routes de pèlerinage, le dévot sait ainsi reconnaître les personnages et scènes sacrés. Les vêtements aux caractères plus locaux paraissent être destinés à des scènes quotidiennes, comme celles liées à l'exercice d'un métier ou à des personnes réelles.

\footnotetext{
${ }^{21}$ Psautier d'Utrecht: Utrecht, Universiteitsbibliotheek, MS Bibl. Rhenotraiectinae I Nr 32, $\mathrm{IX}^{\mathrm{e}}$ siècle. Les copies sont : London, British Library, MS Harley 603 (après l'an Mil), Psautier Eadwine, Cambridge, Trinity College, MS R. 17.1 (1155-1160) et le Psautier Anglo-Catalan, Paris, Bibliothèque nationale de France, Latin 8846 (1180-1200, achevé par un artiste catalan en 1340-1350).

${ }^{22}$ La représentation des figures d'autorité est une vaste question. Néanmoins, un habillement à l'antique ou un vêtement long renvoient à une certaine dignité. Mais cela ne revient pas à dire qu'un personnage ainsi vêtu est forcément positif. Judas et Hérode sont vêtus de la même manière que les autres apôtres ou que des souverains. Car même en tant que personnages négatifs, ils ont un statut précis, en théorie digne. Ce paradoxe des personnages mauvais vêtus comme des personnages exemplaires et respectables fait partie des difficultés de la lecture du costume dans l'art médiéval. Les caractéristiques morales pourront se voir dans les couleurs ou dans les traits du visage.
} 
L'un des meilleurs exemples, à notre sens, est une comparaison entre des pèlerins. Ceux représentés en bas-relief sur la façade de la cathédrale de Fidenza, en Émilie-Romagne, vers 1200, réunissent deux familles, une riche (image 7) et une pauvre. L'une et l'autre sont vêtues selon des conventions antiquisantes. Si nous les comparons à la sculpture dite du «Retour du Croisé » du Musée lorrain à Nancy (image 8), qui présente des personnages ayant réellement existé, la différence est frappante ${ }^{23}$. Le couple de Nancy semble sortir $\mathrm{du} \mathrm{XII}^{\mathrm{e}}$ siècle pour nous raconter son histoire. Les familles de Fidenza sont intemporelles et constituent une invitation à partir en Terre Sainte : Fidenza se trouve sur la Via Francigena, route de pèlerinage pour Rome, d'où, par la Via Appia, l'on pouvait atteindre Brindisi, et de ce port majeur des Pouilles, l'Outremer.

Combien de temps la dimension anachronique est-elle restée perceptible ? Peut-on envisager que le pèlerin médiéval avait conscience du caractère local et daté comme d'une éventuelle règle iconographique? Les vêtements qui ne relèvent pas d'un «anachronique universel», valable dans toute la chrétienté occidentale, sont-ils entendus comme typiquement locaux? Le caractère conventionnel des vêtements des pèlerins anonymes de Fidenza peut être compris comme une métaphore du caractère universel du pèlerinage, d'où l'utilisation de costumes intemporels : cela concerne tous les Chrétiens, quelle que soit leur richesse. La différence de statut est indiquée par des vêtements plus ou moins précieux, selon des principes (décoration, longueur) là aussi compréhensibles dans toute la chrétienté. On peut en retrouver certains détails, sac et bonnet, sur les pèlerins ressuscités et nus du linteau du tympan de la cathédrale d'Autun (image 9), autre église située sur une route de pèlerinage. Ces trois groupes datent de différentes périodes du XII ${ }^{\mathrm{e}}$ siècle. Deux d'entre eux sont intemporels, par la nudité ou le côté antiquisant ; le troisième, celui de

\footnotetext{
${ }^{23}$ Ce groupe sculpté a fait l'objet d'une communication à l'International Medieval Congress de Leeds en 2018, l'étude de son costume permettant de découvrir l'histoire du couple de cette œuvre émouvante.
} 
Nancy, se situe entre les deux précédents, dans le siècle et chronologiquement. Les groupes intemporels sont destinés à servir d'exemples et de signes de ralliement entre pèlerins. Le couple lorrain semble être une statue funéraire ou, à notre avis, un ex-voto, bien plus personnel. Les mêmes signes (bonnet et sac) sont utilisés pour identifier l'activité de pèlerin.

La comparaison avec le groupe sculpté de Nancy montre la différence de costumes entre les pèlerins anonymes, idéalisés, conventionnels et intemporels, d'Autun et Fidenza, et ceux, identifiés et appartenant à leur époque. La sculpture utilisée comme moyen de propagande sur les façades des cathédrales s'oppose à l'expérience personnelle, et c'est le costume séculier qui nous renvoie à l'individualité.

Par ailleurs, certaines scènes quotidiennes, comme les représentations des mois ou des métiers, mélangent parfois passé et présent. Cela paraît compliquer la tâche de l'historien du costume. Mais cette sémiologie prend sens lorsque l'on commence à la maîtriser : quand la lecture de l'image médiévale devient, avec l'expérience, plus aisée, et quand les détails inhabituels interrogent et incitent à développer l'analyse. Apparaissent alors des codes iconographiques qui existent pour eux-mêmes. La richesse du langage de l'image médiévale s'exprime à merveille par l'utilisation faite du costume. Celui-ci habille et pare l'image, lui donne un sens, comme il le fait pour un être humain.

\section{Évoquer un temps révolu ou des espaces lointains}

L'utilisation de costumes anachroniques est une convention iconographique pour certains personnages sacrés, comme le Christ ou les apôtres, qui sont traditionnellement vêtus à l'antique et parés de tuniques, de toges ou de sandales. Ces éléments servent à renvoyer l'observateur au passé, à l'autorité, à la dignité. Les costumes à l'antique peuvent ainsi vêtir héros et héroïnes bibliques ou mythologiques, personnages historiques, mais aussi figures allégoriques. 
Mais en dehors de cet usage classique et facilement intelligible, l'anachronisme du costume sert également à marquer le caractère exotique du personnage représenté et correspond souvent aux conventions graphiques établies dans l'Antiquité pour représenter l'étranger : l'abondante décoration des vêtements ou les bonnets phrygiens, qui distinguaient les Orientaux, désignent au Moyen Âge les non-Chrétiens ou des étrangers, selon des modalités très élaborées ${ }^{24}$. En effet, dans l'iconographie médiévale occidentale, plus un personnage est proche - à la fois géographiquement, temporellement ou religieusement $-\mathrm{du}$ contexte culturel dans lequel évolue l'artiste, plus son costume sera proche du quotidien d'alors. Plus il est éloigné, plus sa tenue sera étrange. Il est ainsi bien vain de chercher une quelconque réalité historique à ces costumes. Les enlumineurs et artistes, représentant des pièces de costumes diverses et hétéroclites, ont créé une véritable sémiologie graphique purement occidentale. Ce sont apparemment des anachronismes de fantaisie ne relevant pas d'une étude scientifique des objets antiques, à de rares exceptions près, généralement italiennes et très souvent tardives (à partir du XIII siècle) ${ }^{25}$. Pourtant, ces étranges objets ont souvent une origine ancienne, déformée par le temps. Le résultat en est parfois fantaisiste et surprenant. Ainsi des bourreaux de saints sont-ils affublés d'ailes sur leur tête dans des enluminures parisiennes du $\mathrm{XIV}^{\mathrm{e}}$ siècle $^{26}$. On peut supposer sans trop de risque qu'il s'agit d'une mauvaise compréhension des représentations des casques affublant Hermès, Hadès ou

\footnotetext{
${ }^{24}$ Voir Tina Anderlini, «L'évolution des signes de judéité par les coiffes dans l'image à la fin du Moyen Âge »(titre provisoire), dans Vêtements, costumes et religion. Actes du colloque tenu à Nancy les 28 et 29 septembre 2017, Rennes, Presses universitaires de Rennes, à paraître.

${ }^{25}$ On pense évidemment en premier lieu au buste de Frédéric II en empereur romain, ainsi qu'à d'autres objets liés à l'empereur Staufen, provenant directement de l'art antique. On pense aussi à tous les sculpteurs annonciateurs de la Renaissance comme Pisano, dans la cathédrale de Pise. Toujours à Pise, on remarque les très belles armures antiquisantes des anges du Jugement Dernier du Campo Santo. Ce sont d'ailleurs bien souvent les éléments militaires qui sont les plus notables.

${ }^{26}$ Jeanne de Montbaston, Vies de saints, «Saint Paul prêchant», $2^{\mathrm{e}}$ quart du XIV siècle, Bibliothèque nationale de France, fr. 185, fol. 34.
} 
Persée, casques venant du passé et renvoyant celui qui les regarde au passé par leur aspect insolite. La rupture visuelle avec le quotidien du spectateur devient le signal du passage à une autre époque.

Mais en s'approchant de la fin du Moyen Âge, on commence à trouver des pièces d'armement antiques mêlées aux armures de plates. Piero della Francesca en est un bon exemple. Dans sa célèbre fresque d'Arezzo, «La Victoire d'Héraclius sur Chosroès ${ }^{27} \gg$ » (image 10), le peintre mélange la quasinudité antique et les armures romaines avec des détails qui lui sont contemporains, comme ces fameuses armures de plates ou des chausses et doublets, parmi d'autres vêtements à la mode au milieu du Quattrocento. Piero ajoute même, pour que l'on identifie l'armée impériale, l'Aigle de l'Empire Romain Germanique, anachronisme indispensable pour reconnaître le camp chrétien, alors que les païens se battent sous la bannière de la tête de Maure. Passé et présent rendent la fresque compréhensible. La lutte du Saint Empire contre les Maures permettait-elle aussi au spectateur de s'identifier aux défenseurs de la Chrétienté, alors que Constantinople venait de tomber? L'éloignement temporel est réel, mais le spectateur a la possibilité d'identifier les deux camps et, peut-être, de s'y reconnaître. Si le Quattrocento montre un souci de réalisme, ce dernier ne doit pas empêcher la bonne compréhension de l'œuvre.

La sculpture antique inspire régulièrement la sculpture médiévale, mais l'introduction des éléments métalliques romains travestissant des costumes médiévaux est une nouveauté de la fin du Moyen Âge. Ces détails portent en plus parfois la marque du temps : verdis comme on peut les voir à Arezzo, ils renvoient aux pièces en métal retrouvées, collectionnées par les amateurs

${ }^{27}$ Arezzo, Basilique Saint-François, vers 1460. 
d'antique, et étudiées par des artistes comme Piero ou encore Andrea Mantegna ${ }^{28}$.

Ces mélanges insolites permettaient aux contemporains de l'œuvre d'en saisir immédiatement le sens : la bataille est aussitôt identifiée grâce aux armures des hommes. Mais c'est aussi une bataille d'un lointain passé, ce décalage temporel étant identifiable grâce aux éléments militaires ou vestimentaires inhabituels. Les érudits de l'époque, eux, pouvaient apprécier les connaissances de l'artiste. Ce mélange d'époques paraît jeter un pont entre deux périodes historiques. Le péril païen, combattu par Héraclius, est toujours présent. Peut-être ces allers-retours continuels entre passé et présent ont-ils un sens bien plus profond que notre propre éloignement temporel avec leur époque de création, sens qu'une tendance à oublier le contexte historique autour des œuvres ne nous fait pas toujours percevoir.

\section{Double anachronisme : des costumes anciens sans cesse réactualisés}

Ces mélanges d'anachronisme et de modernité compliquent, comme nous l'avons déjà signalé, l'analyse du costume pour les historiens. Les saints et saintes suivent souvent la mode. Doit-on y lire une volonté d'en faire des exemples, incitant les bons Chrétiens à les imiter, le costume favorisant l'identification? La Vierge elle-même finit par s'adapter à la mode de l'époque où elle est représentée, cette sécularisation de Marie semblant s'arrêter net avec la Contre-Réforme. La tradition, passant par le vêtement, deviendrait-elle un refuge?

On assiste, au cours du Moyen Âge, à une paradoxale sécularisation de l'anachronisme. On garde les principes du costume traditionnel, tout en mettant plus ou moins celui-ci à la mode. C'est souvent le cas des tenues féminines. On

\footnotetext{
${ }^{28}$ Mantegna (1431-1506) s'est d'ailleurs représenté en soldat romain dans l'une des fresques (détruites) de l'église des Eremitani à Padoue. Il est remarquable par son étude de l'art romain visible dans grand nombre de ses œuvres, que ce soit pour les costumes militaires, les bâtiments ou les accessoires divers.
} 
conserve de certaines traditions paléochrétiennes une tunique plus courte, portée sur une tunique blanche couvrant les pieds. Mais si cette tunique du dessus a longtemps eu une allure antique, elle peut aussi avoir une forme plus moderne. L'anachronisme est alors situé dans la vision de cette tunique blanche, le costume médiéval ne permettant cela qu'en montrant les sous-vêtements, ce qui serait indécent ${ }^{29}$.

Un cas étonnant se trouve dans le Roman de la Poire ${ }^{30}$. Le costume appartient au XIII ${ }^{\mathrm{e}}$ siècle, mais la tunique blanche, visible lorsque Pitié lève sa robe (image 11), renvoie à la tradition. Ce vêtement pourrait être pris pour une chemise, mais il est trop long par rapport aux sous-vêtements du temps et il est surtout inconvenant de la montrer, particulièrement pour une allégorie positive. Car chaque détail qui semble original doit être appréhendé avec d'infimes précautions, et encore et toujours contextualisé. Si un élément n'apparaît que sur des personnages du passé (réels ou imaginaires), ou des personnages exotiques - qui constituent hélas la grande majorité des personnages de l'art médiéval -, il convient d'avoir une analyse des plus rigoureuses. Et même si certains saints, héros ou autres paraissent vêtus selon les règles de l'époque de création de l'œuvre, on remarquera qu'un détail qui relève du passé, de l'exotisme ou d'une fantaisie vient, de temps à autre, se mêler aux éléments contemporains. Pitié est d'ailleurs la seule allégorie du Roman de la Poire gardant une trace de l'iconographie antique. Le texte n'explique en rien cette particularité. Il ne semble pas qu'une règle stricte ou une séquence d'apparition puisse être établie. Ces détails agissent comme des rappels de contexte et surgissent au gré de l'auteur.

Mais comme nous sommes devant des œuvres médiévales, nous allons aussi croiser, parfois, des personnages du passé qui portent les tenues les plus représentatives de la date de création de l'œuvre. Ces individus restent pourtant

\footnotetext{
${ }^{29}$ La chemise commence seulement à se montrer sans honte au cours du XV $\mathrm{XV}^{\mathrm{e}}$ siècle.

${ }^{30}$ Paris, Bibliothèque nationale de France, fr. $2186,3^{\mathrm{e}}$ quart du XIII ${ }^{\mathrm{e}}$ siècle.
} 
fort rares, et une étude de toute l'œuvre qui les comprend peut identifier un élément archaïsant dans un accessoire, dans le décor, ou dans le paysage. L'altérité, quelle qu'elle soit, est mise en scène de bien des manières.

On peut donc constater que l'importance sociale du costume au Moyen Âge en fait un élément fondamental dans l'art. Le costume est le vecteur idéal lorsque l'on veut préciser le statut social, la respectabilité ou l'éloignement spatio-temporel d'un personnage. Sainte Catherine, épouse mystique du Christ, est habillée comme une princesse de la date de création de l'œuvre, mais plus richement encore; Salomé est aussi à la mode, parfois trop selon les tolérances de l'Église, avec des détails exotiques (rappelant, là encore, l'abondance d'ornementation que l'on note sur sainte Catherine). À côté de ces saintes et figures bibliques coquettes, dont la liste est loin d'être exhaustive, alliant modernité et tradition, d'autres personnages semblent vêtus de manière intemporelle. C'est le cas de saint Jean-Baptiste, ermite et cousin du Christ, portant la pauvre mélote et le riche manteau. Ce mélange des époques, du temporel et de l'intemporel, a déjà été mis en évidence par Jean Wirth, qui rappelle avec justesse les difficultés à lire ces images :

Il arrive que des images reproduisent avec exactitude les objets en usage, mais c'est loin d'être toujours le cas. Le piège est particulièrement dangereux pour l'historien du costume, entre autres parce que le goût de l'exotisme multiplie les vêtements de fantaisie, tandis que les personnages principaux de l'iconographie religieuse, le Christ et les apôtres, n'ont guère changé de tenue depuis la basse Antiquité ${ }^{31}$.

Ainsi, l'iconographie médiévale use de costumes obsolètes, désuets ou anachroniques en donnant à chacun une signification qui lui est propre. Le plus dévalorisant paraît être l'obsolète ; le plus valorisant, le désuet, garant d'une tradition séculaire et marque d'honorabilité. L'anachronisme apparaît a priori

\footnotetext{
${ }^{31}$ Jean Wirth, L'image du corps au Moyen Âge, Firenze, Sismel-Edizioni del Galluzzo, 2013, p. 7.
} 
comme plus neutre et peut résulter d'une méconnaissance du passé. Mais cela n'empêche pas qu'il soit parfois utilisé pour préciser le caractère négatif, ou du moins «étranger au bon Chrétien », de celui qui est justement vêtu étrangement. L'exemple d'Arezzo nous montre que costumes et autres symboles textiles entremêlant les siècles tissent peut-être des liens entre deux époques et deux lieux éloignés, en rappelant la présence d'un même danger. Considérant la nécessité de contextualiser systématiquement une œuvre médiévale, on trouvera naturellement des exemples de costumes désuets et dévalorisants, comme de costumes obsolètes et valorisants. Une constatation s'impose : la connaissance du costume médiéval, en progrès constant, tend à devenir une clef de lecture de plus en plus fondamentale du caractère symbolique des œuvres du Moyen Âge. Cette connaissance permet d'avancer ou de rejeter des hypothèses et des interprétations. Le costume n'était pas représenté par les artistes médiévaux, mais utilisé; il leur permettait ainsi de préciser leur propos. Il est un outil iconographique, semblable au lys signifiant la virginité de Marie, à la pomme rappelant le Péché Originel. Et pour connaître ce costume, il faut donc, comme nous l'avons vu, savoir faire un tri raisonné dans la garde-robe. Il convient de reconnaître ce qui vient du passé, ce qui est emprunté aux espaces extraeuropéens, ce qui vient de la tradition iconographique. Ces détails nous permettent de comprendre un peu mieux la société médiévale et ses rapports à l'altérité. Mais savoir identifier et appréhender ces éléments nous aide aussi à repérer des restaurations, et même à dater les œuvres d'art. À condition d'éviter certains écueils, la mode, symbole de la superficialité, devient un élément primordial pour l'histoire de l'art médiéval. 


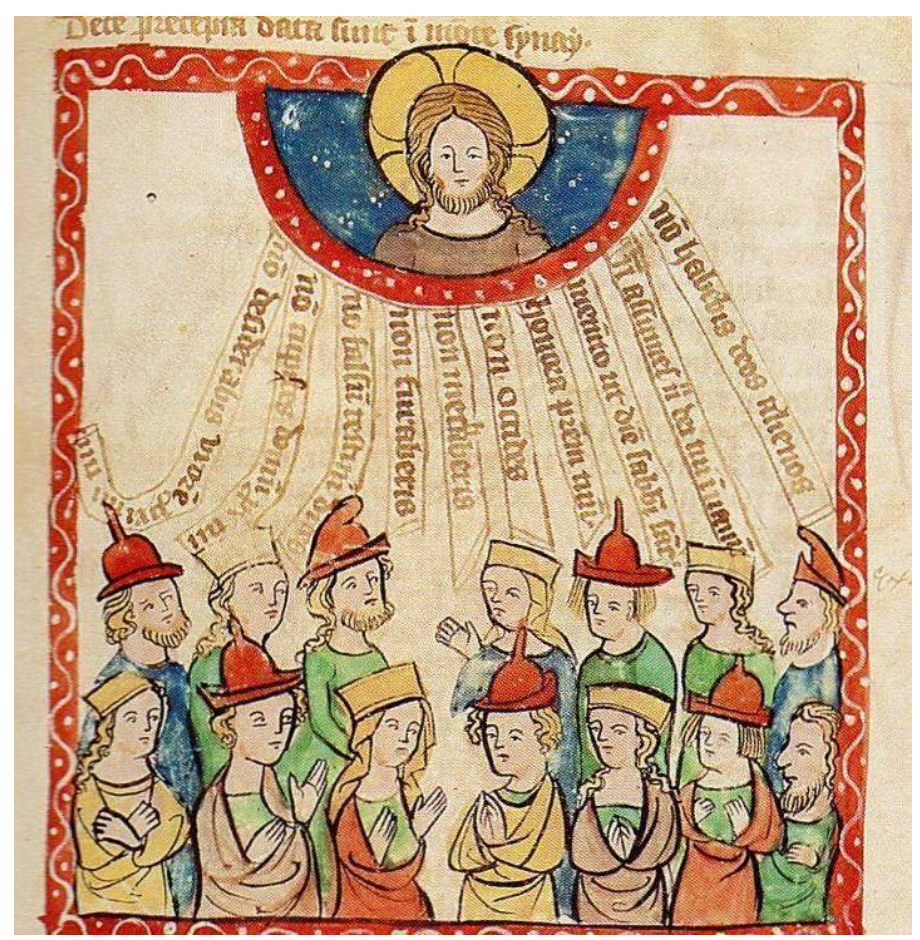

Image 1 : Les Juifs recevant les Dix Commandements, Heilsspiegel, vers 1360, Landesbibliothek Darmstadt, Ms 2505, chap. 32 (34) (Photo Wikimedia Commons)

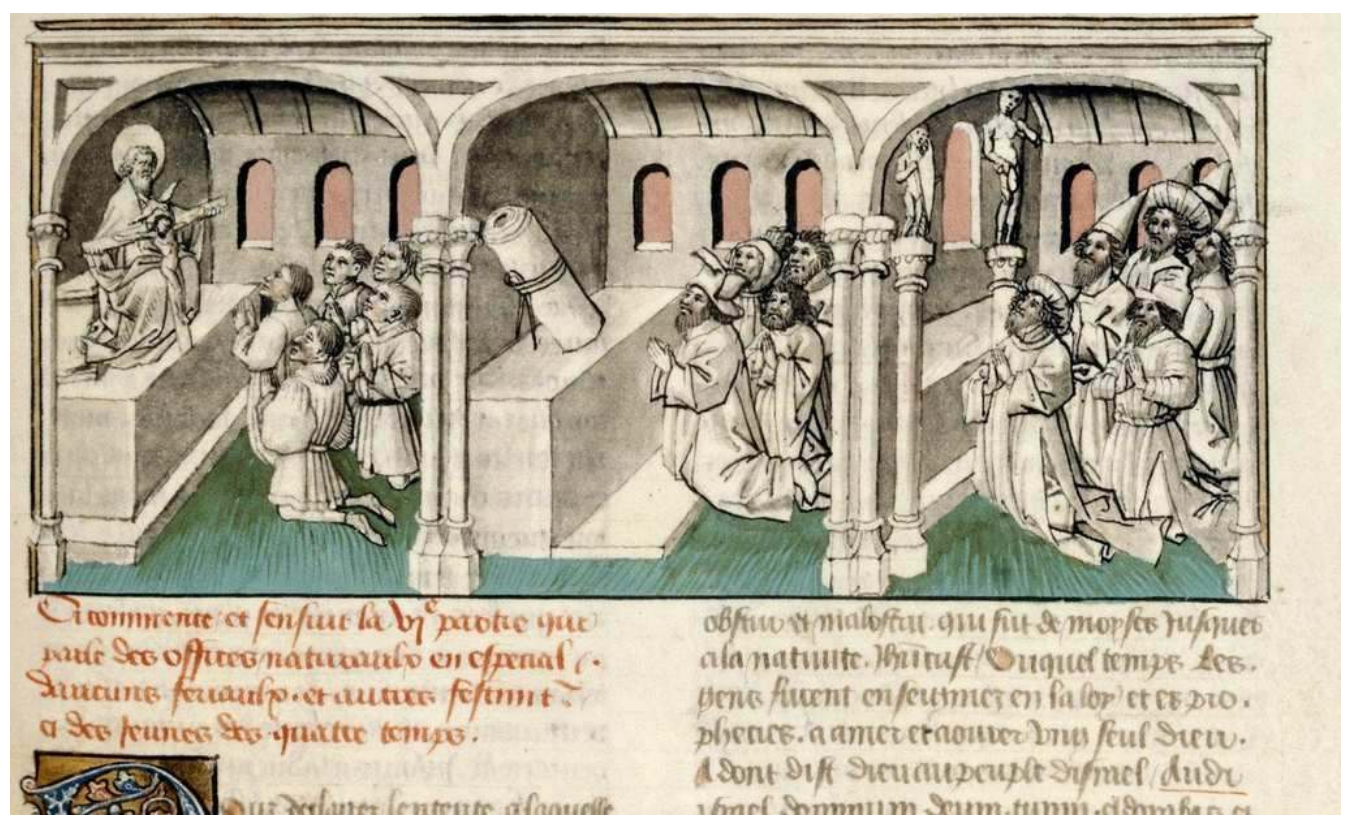

Image 2 : Rational des divins offices, début du $\mathrm{Xv}^{\mathrm{e}}$ siècle, Bibliothèque municipale, Beaune, ms 21, f. 149 (Photo $\odot$ Bibliothèque de Beaune) 


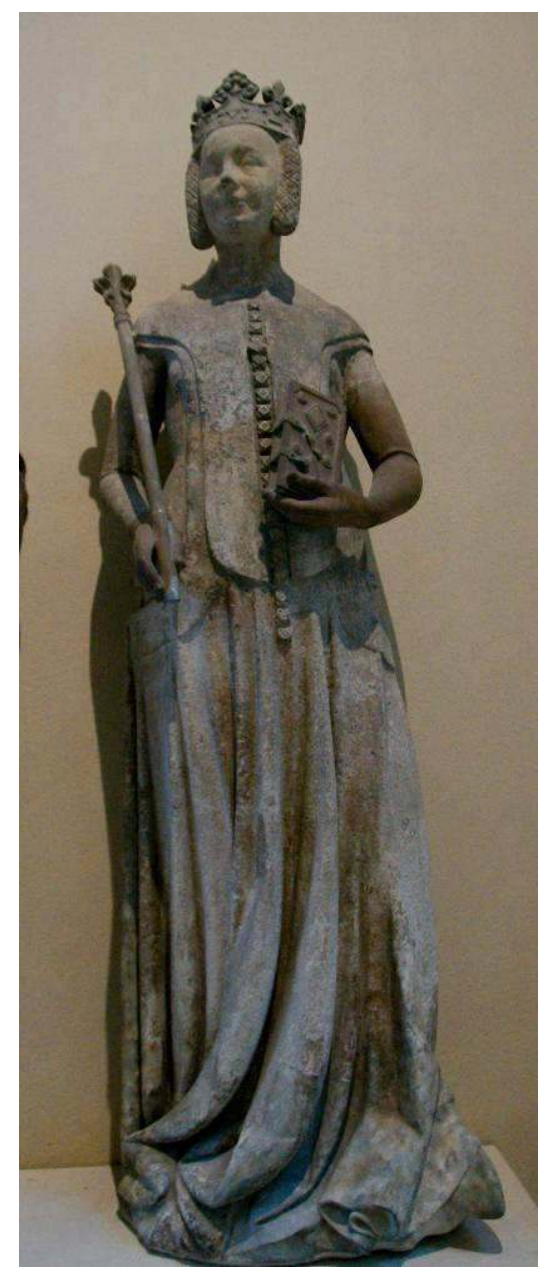

Image 3 : Jeanne de Bourbon, 1365-1380, Musée du Louvre, Paris (Photo T. A.)

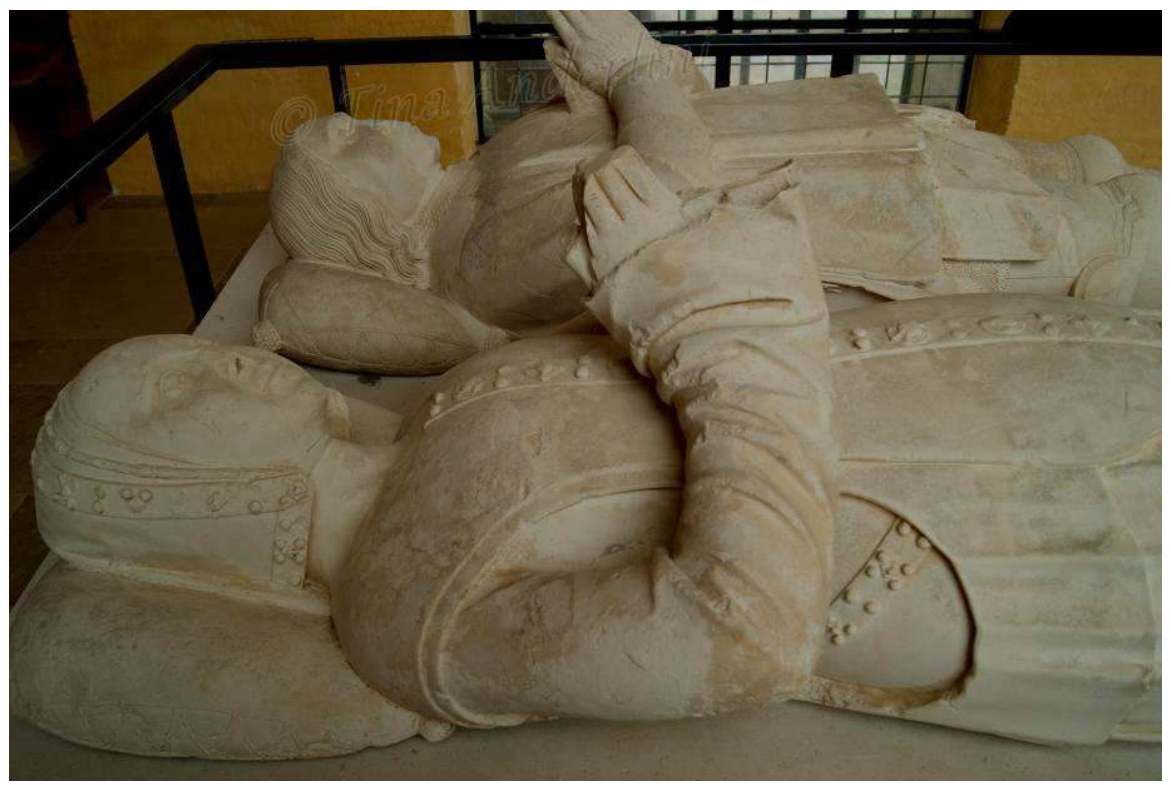

Image 4 : Gisant de Jeanne de Hangest, morte en 1514, Musée Antoine Vivenel, Cloître Saint-Corneille, Compiègne. (Photo T. A.) 


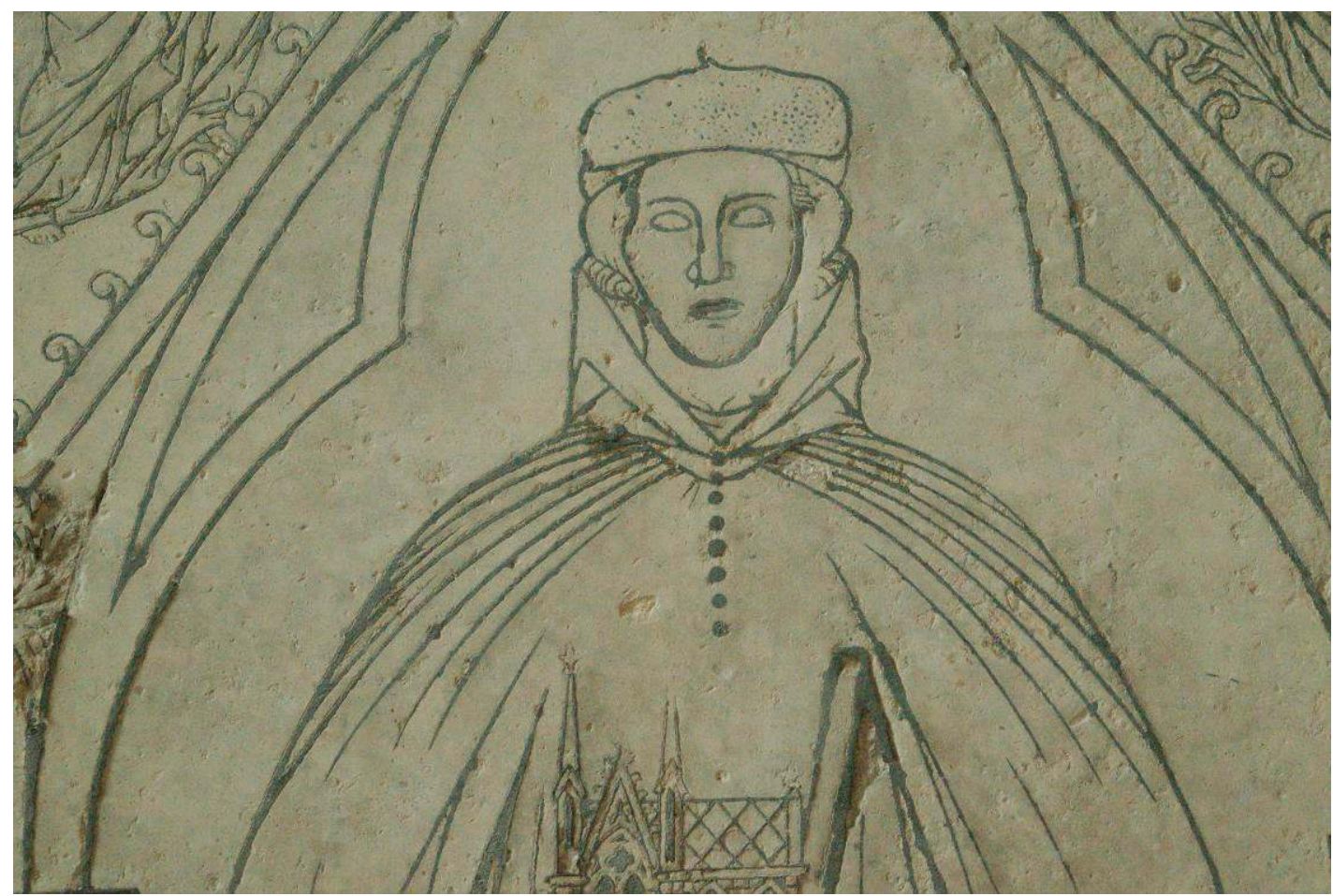

Image 5 : Plaque tombale de l'architecte Hugues Libergier, mort en 1263, provenant de l'église Saint-Nicaise, cathédrale de Reims (Photo T. A.)

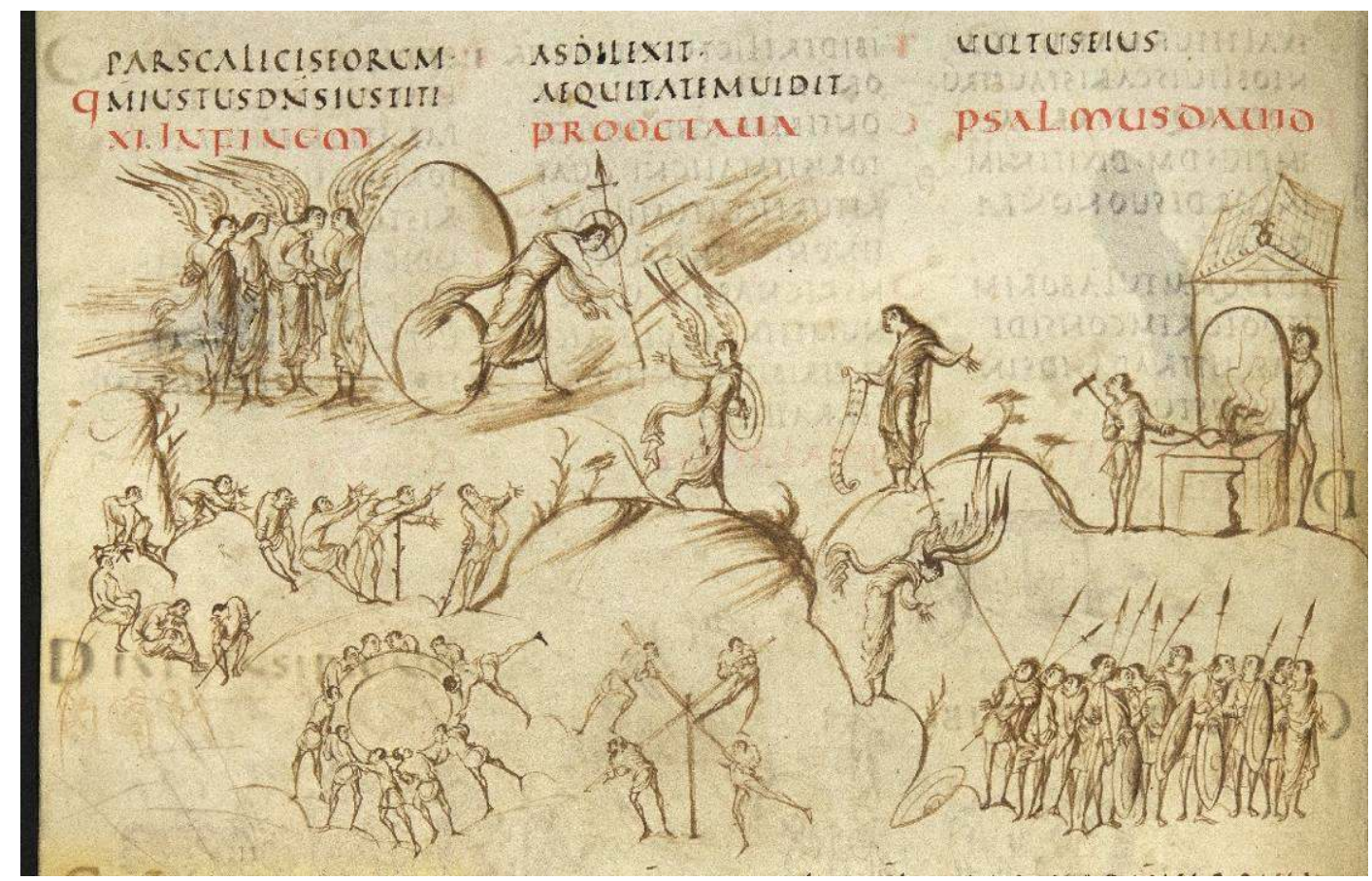

Image 6 : École de Reims, Psautier d'Utrecht, Psaume 11, IX ${ }^{\mathrm{e}}$ siècle, Utrecht, Universiteitsbibliotheek, MS Bibl. Rhenotraiectinae I Nr 32, f. 6v (Photo Wikimedia Commons) 


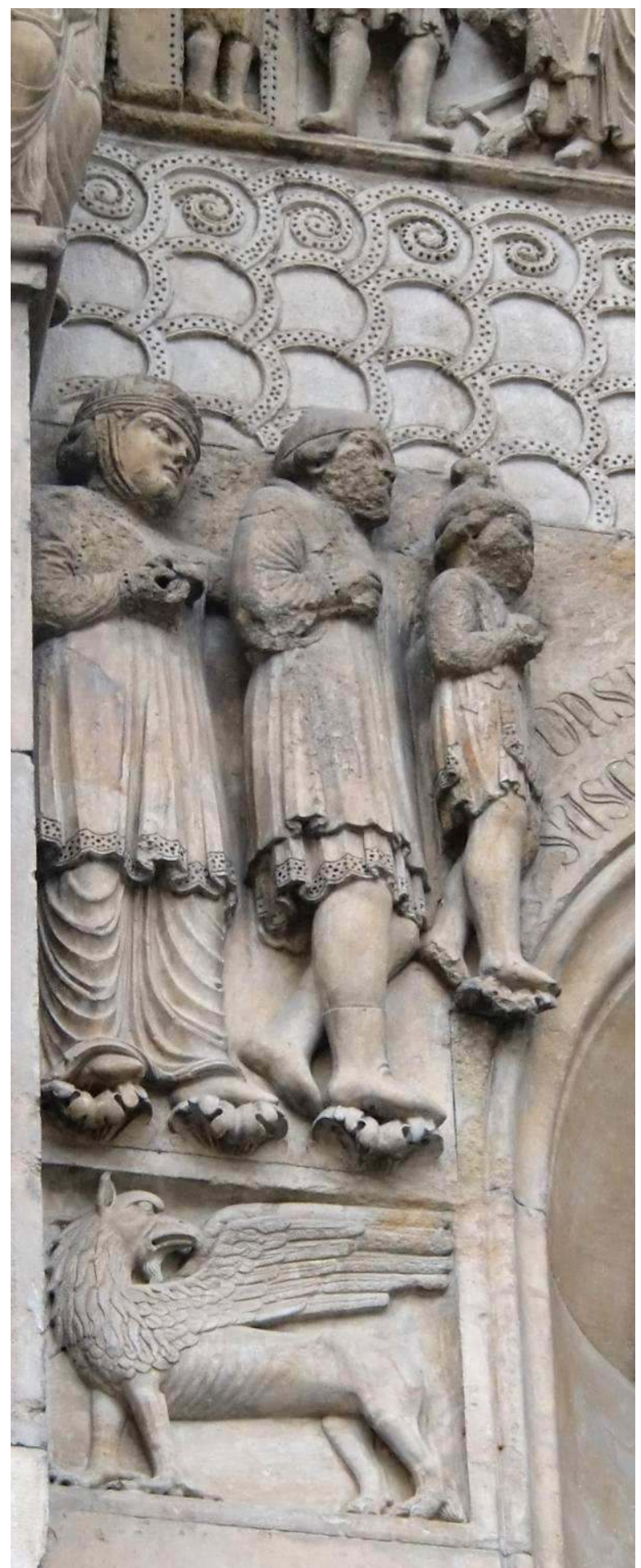

Image 7 : Atelier de Benedetto Antelami. Les Riches Pèlerins, autour de 1200, cathédrale, Fidenza. (Photo Laurom, Wikimedia Commons) 


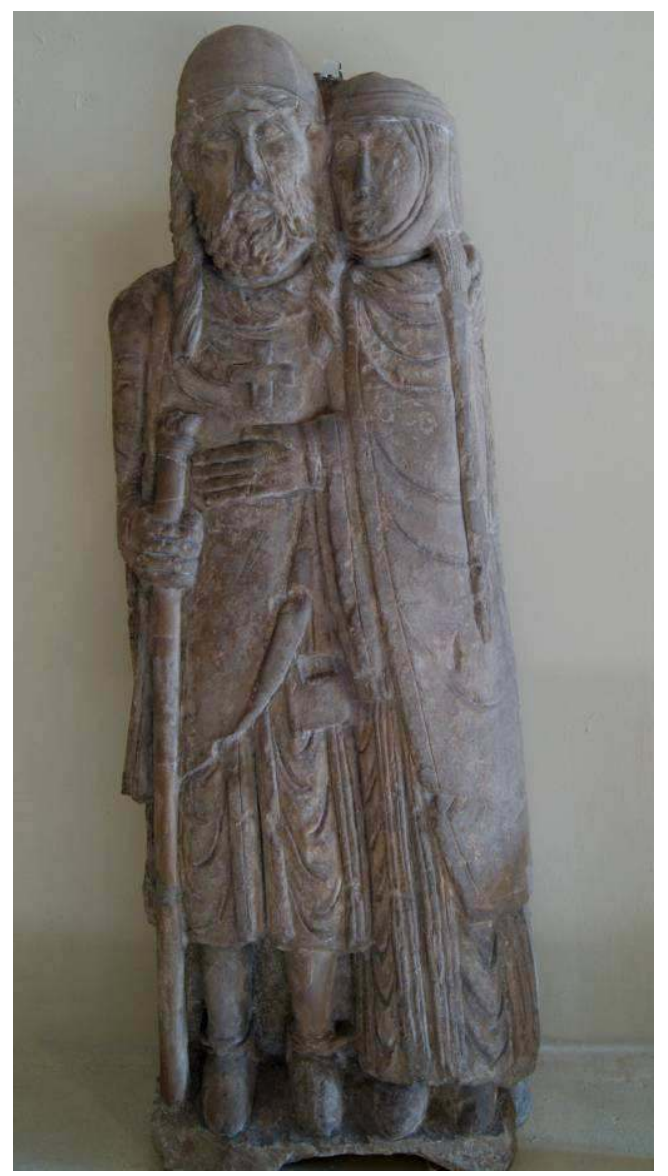

Image 8 : Le Retour du Croisé, fin du XII ${ }^{e}$ siècle, Musée lorrain, Nancy (Photo T. A.)

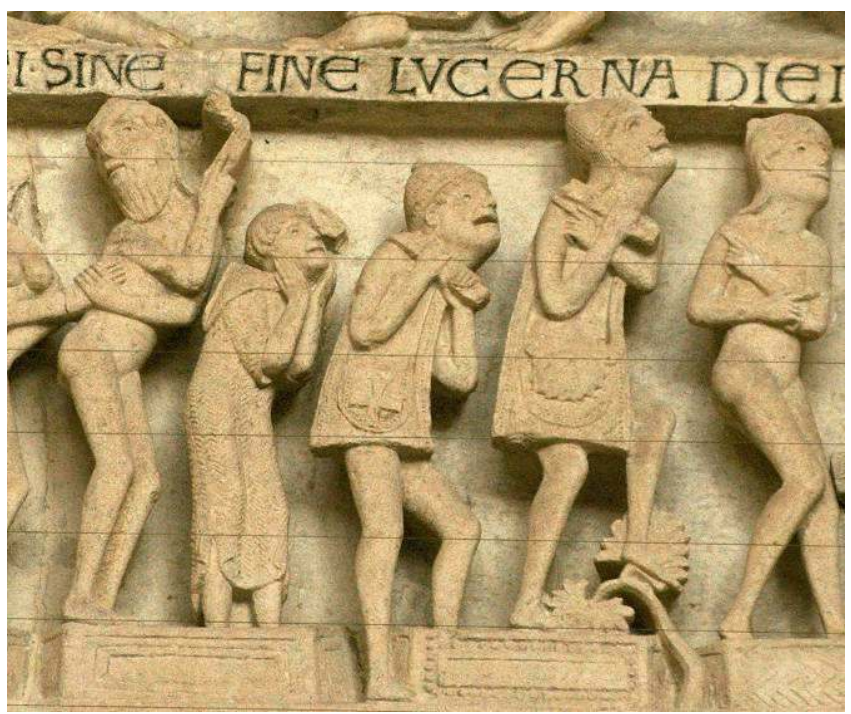

Image 9 : Gislebertus (?), vers 1130-1135, Pèlerins du Jugement Dernier, cathédrale d'Autun (Photo T. A.) 


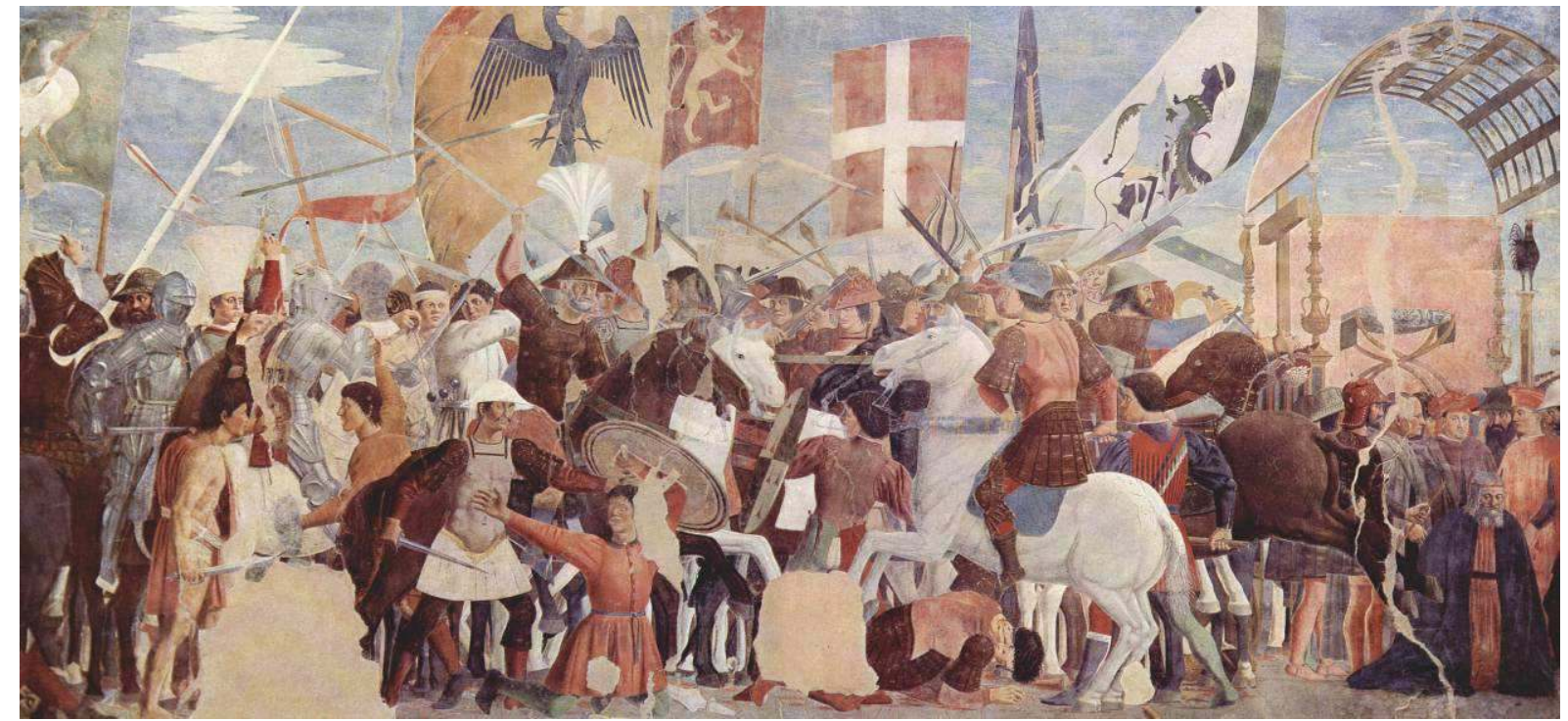

Image 10 : Piero della Francesca, Victoire d'Héraclius sur Chosroès, 1456-1462, basilique SaintFrançois, Arezzo (Photo Wikimedia Commons)

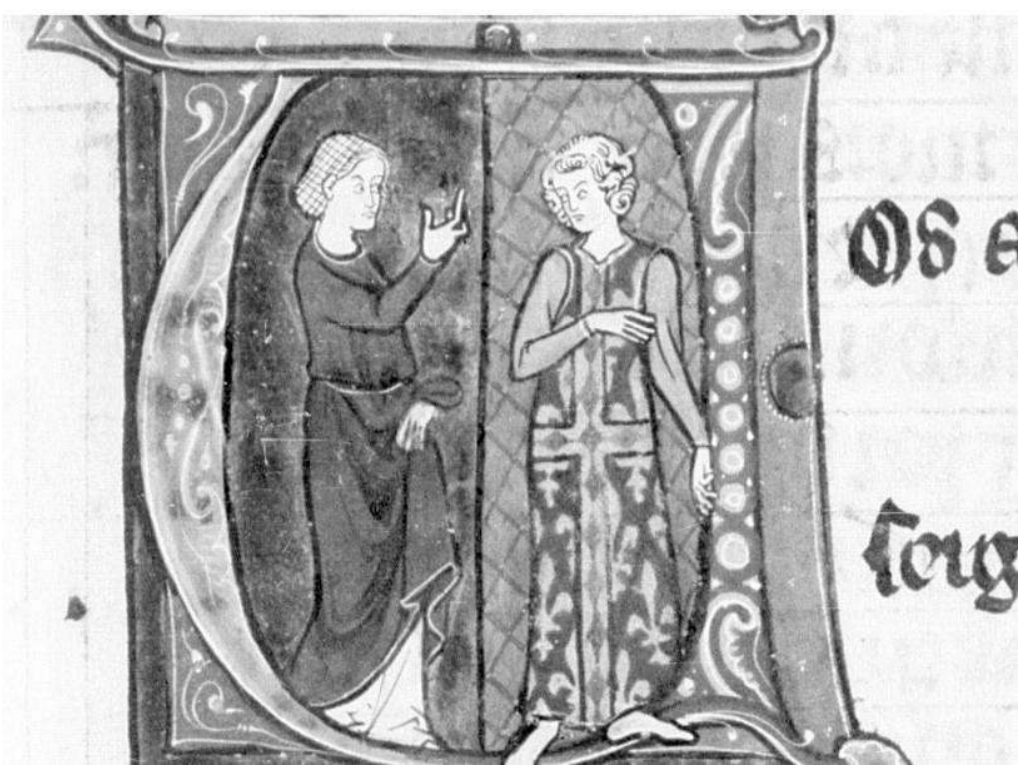

Image 11 : Le Roman de la Poire, Pitié ou Humilité, $3^{\mathrm{e}}$ quart du XIII ${ }^{\mathrm{e}}$ siècle, Bibliothèque Nationale de France, MS Français 2186, f. 71 (Photo OBibliothèque Nationale de France) 
